\title{
A Case Report: Spontaneous Pneumomediastinum (Hamman's Syndrome)
}

\author{
Aakash N. Bodhit ${ }^{\mathrm{a}, \mathrm{b}}$, Latha G. Stead ${ }^{\mathrm{a}}$
}

\begin{abstract}
Spontaneous pnemomediastinum is a rare medical condition which usually occurs in young male patients with a history of inhalational drug use. Chest pain and dyspnea are common presenting symptoms, and precordial crunching sound on auscultation synchronous with heartbeats is a characteristic sign of this condition. Also known as Hamman's syndrome, it is usually self- limiting and without any complication or recurrence.
\end{abstract}

Keywords: Spontaneous pnemomediastinum; Precordial crunching; Hamman's syndrome

\section{Introduction}

Spontaneous pneumomediastinum is not so common medi-

Manuscript accepted for publication January 27, 2012

\footnotetext{
${ }^{a}$ Department of Emergency Medicine, University of Florida College of Medicine, Gainesville, FL 32610, United States

${ }^{b}$ Corresponding author: Aakash N. Bodhit, Department of Emergency Medicine,University of Florida College of Medicine, 1329 SW 16th St, PO Box 100186, Gainesville, FL 32610-0186, United States. Email: aakashnb@ufl.edu
}

doi:10.4021/jmc530w cal condition seen in the Emergency Medicine department. It is estimated to be present in approximately 1 in 30,000 Emergency Department referrals [1]. Though it is a benign and self-limiting condition, it often leads to a number of investigations due to possibility of a potentially serious condition such as a rupture of any of the viscera or simply because of misdiagnosis. It rarely leads to any complication.

The condition was described by Louis Hamman and hence it is also known as Hamman's syndrome.

\section{Case Report}

A15 year old male patient was referred to our Emergency department, which is a part of an academic tertiary care medical facility. The referral was for the evaluation of subcutaneous emphysema.

The patient had presented with dull resting chest pain, which was gradual in onset. After an hour of dull pain, the

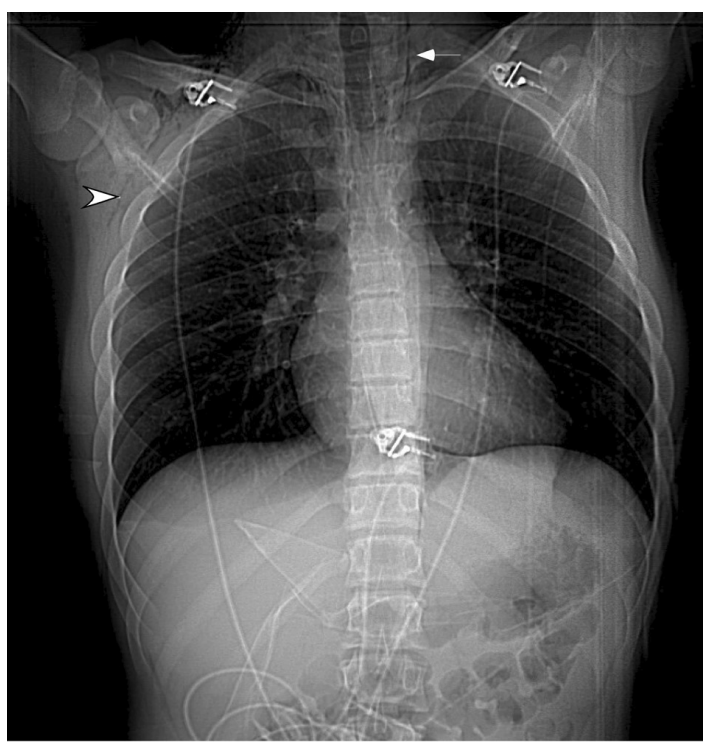

Figure 1. Chest $x$ - ray (scout view) depicting lucency along left mediastinal border (arrow) indicating pneumomediastinum. Also note the subcutaneous emphysema on the right side (arrow head). 


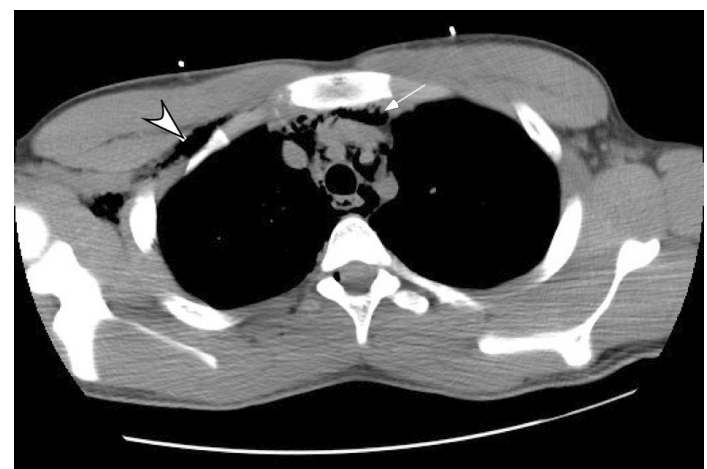

Figure 2. CT Chest confirming subcutaneous emphysema (arrow head) and extensive pneumomediastinum (arrow)

nature of the pain changed to sharp with radiation to his throat. The patient went to an outside Emergency Room (ER) where he was evaluated for his chest pain. Vital signs were found to be within normal limits. Physical examination was unremarkable. Routine laboratory evaluations such as $\mathrm{CBC}$, and Basic Metabolic Panel (BMP) revealed no abnormality. A chest $\mathrm{X}$ - ray examination was remarkable for presence of subcutaneous emphysema (Fig. 1). He was subsequently transferred to our Emergency department without any further intervention.

Patient was symptom free upon arrival to emergency department of our hospital. He denied any history of any trauma, foreign body ingestion or prior history of such episodes. The vital signs were normal. General examination revealed well appearing male, who was alert, oriented, and in no acute distress. On systemic physical examination, palpable subcutaneous emphysema over the right trapezius muscle and neck was present. There were no abnormal auscultatory findings on respiratory and cardiac examination. The 12- lead electrocardiogram was without any rhythm or repolerization abnormalities. A CT scan of the chest was obtained.

The CT scan of the Chest confirmed the presence of subcutaneous emphysema as seen in the Fig. 2 (arrow ahead). There was additional evidence of extensive pneumomediastinum (arrow in Fig. 2). There was no pneumothorax present. Also, no obvious esophageal or tracheolaryngeal perforation or fistula was present. After ruling out all other conditions, he was diagnosed as having Hamman's syndrome. Patient was put under medical observation and monitored for a day and subsequently discharged to home without worsening of condition or any complications. Patient was followed up four days later and his emphysema was completely resolved by then. A chest $\mathrm{x}$ - ray on follow- up visit was normal. His subsequent clinical course was normal.

\section{Discussion}

Spontaneous pneumomediastinum (Hamman's syndrome) is a rare condition which is usually benign and non recurrent. It is known to be associated with transiently elevated intra alveolar pressure. Other causes of pneumomediastinum such as trauma (blunt or penetrating trauma to chest), forceful vomiting (Boerhaave's syndrome), medical procedures such esophagoscopy, bronchoscopy should be ruled out.

In three retrospective analyses, it has been noted that young male patients are affected more often by this condition and it is associated with inhalational drug use [1-3]. It should be noted though that it has also been reported in women in the absence of inhalational drug use [4].

The most common presentation is chest pain and dyspnea, with subcutaneous emphysema being the most common sign. Palpable crepitus may be absent however [4]. Chest pain is usually retrosternal and it may radiate to neck or into the back. In almost all patients, vital signs are normal and they appear well and healthy. Precordial crunching sound synchronous with heartbeat is the characteristic sign, which is called Hamman's sign. It is present in more than half the patients, though it was absent in this case.

Treatment is expectant. Medical observation of a few hours or few days are required depending upon the patient symptoms and signs. The patients can be discharged safely as typically they do not develop any complications, nor do they have recurrence.

\section{Conflict of Interest}

Both authors declare no conflict of interest.

\section{References}

1. Newcomb AE, Clarke CP. Spontaneous pneumomediastinum: a benign curiosity or a significant problem? Chest. 2005;128(5):3298-3302.

2. Panacek EA, Singer AJ, Sherman BW, Prescott A, Rutherford WF. Spontaneous pneumomediastinum: clinical and natural history. Ann Emerg Med. 1992;21(10):12221227.

3. Kelly S, Hughes S, Nixon S, Paterson-Brown S. Spontaneous pneumomediastinum (Hamman's syndrome). Surgeon. 2010;8(2):63-66.

4. Mohseni MM. Spontaneous pneumomediastinum. Int J Emerg Med. 2008;1(3):229-230. 